\title{
Direct comparison of the acute effects of lysergic acid diethylamide and psilocybin in a double-blind placebo-controlled study in healthy subjects
}

\author{
Friederike Holze ID $^{1,2}$, Laura Ley ${ }^{1,2}$, Felix Müller ${ }^{3}$, Anna M. Becker ${ }^{1,2}$, Isabelle Straumann ${ }^{1,2}$, Patrick Vizeli (iD ${ }^{1,2}$, Sebastian Silva Kuehne ${ }^{1,2}$, \\ Marc A. Roder ${ }^{1,2}$, Urs Duthaler (D) ${ }^{1,2}$, Karolina E. Kolaczynska ${ }^{1,2}$, Nimmy Varghese ${ }^{3,4}$, Anne Eckert (iD) ${ }^{3,4}$ and Matthias E. Liechti (iD ${ }^{1,2}{ }^{\bowtie}$
}

(c) The Author(s) 2022

\begin{abstract}
Growing interest has been seen in using lysergic acid diethylamide (LSD) and psilocybin in psychiatric research and therapy. However, no modern studies have evaluated differences in subjective and autonomic effects of LSD and psilocybin or their similarities and dose equivalence. We used a double-blind, randomized, placebo-controlled, crossover design in 28 healthy subjects ( 14 women, $14 \mathrm{men}$ ) who underwent five $25 \mathrm{~h}$ sessions and received placebo, LSD (100 and $200 \mu \mathrm{g}$ ), and psilocybin (15 and $30 \mathrm{mg}$ ). Test days were separated by at least 10 days. Outcome measures included self-rating scales for subjective effects, autonomic effects, adverse effects, effect durations, plasma levels of brain-derived neurotrophic factor (BDNF), prolactin, cortisol, and oxytocin, and pharmacokinetics. The doses of 100 and $200 \mu \mathrm{g}$ LSD and $30 \mathrm{mg}$ psilocybin produced comparable subjective effects. The $15 \mathrm{mg}$ psilocybin dose produced clearly weaker subjective effects compared with both doses of LSD and $30 \mathrm{mg}$ psilocybin. The $200 \mu \mathrm{g}$ dose of LSD induced higher ratings of ego-dissolution, impairments in control and cognition, and anxiety than the $100 \mu \mathrm{g}$ dose. The $200 \mu \mathrm{g}$ dose of LSD increased only ratings of ineffability significantly more than $30 \mathrm{mg}$ psilocybin. LSD at both doses had clearly longer effect durations than psilocybin. Psilocybin increased blood pressure more than LSD, whereas LSD increased heart rate more than psilocybin. However, both LSD and psilocybin showed comparable cardiostimulant properties, assessed by the rate-pressure product. Both LSD and psilocybin had dose-proportional pharmacokinetics and first-order elimination. Both doses of LSD and the high dose of psilocybin produced qualitatively and quantitatively very similar subjective effects, indicating that alterations of mind that are induced by LSD and psilocybin do not differ beyond the effect duration. Any differences between LSD and psilocybin are dose-dependent rather than substance-dependent. However, LSD and psilocybin differentially increased heart rate and blood pressure. These results may assist with dose finding for future psychedelic research.
\end{abstract}

Trial registration: ClinicalTrials.gov identifier: NCT03604744

Neuropsychopharmacology (2022) 47:1180-1187; https://doi.org/10.1038/s41386-022-01297-2

\section{INTRODUCTION}

Lysergic acid diethylamide (LSD) and psilocybin are both classic serotonergic psychedelics that are used recreationally [1] and have recently become promising candidates for the treatment of various psychiatric disorders (e.g., anxiety disorders and major depressive disorder) and neurologic disorders (e.g., cluster headache and migraine) [2-6]. Both substances induce complex alterations of mind via stimulation of the serotonin 5hydroxytryptamine-2A $\left(5-\mathrm{HT}_{2 \mathrm{~A}}\right)$ receptor [7-9]. LSD exerts additional activity at dopamine $\mathrm{D}_{1-3}$ receptors, whereas psilocin, the active metabolite of psilocybin, inhibits the serotonin transporter [10]. Whether these differences in receptor binding profiles produce differential subjective effects in humans has not yet been studied. Recent research has investigated either psilocybin or LSD alone [7, 11-15]. Differences between the two substances with regard to their acute effects, similarities, and doseequivalence remain unclear.

Therefore, the present study evaluated and directly compared the acute subjective, autonomic, and endocrine effects of LSD and psilocybin using two doses of each substance and placebo within the same subjects. The acute subjective effects of LSD and psilocybin were determined using validated psychometric instruments that are used internationally in healthy subjects as well as in therapeutic studies in patients [3, 16-18]. In addition, we determined plasma LSD and psilocin concentrations over time and over $24 \mathrm{~h}$ to provide valid pharmacokinetic parameters for all substances and doses used. Previous research has shown that plasma concentrations of LSD and psilocybin are strongly linked to subjective effects $[7,19,20]$. Pharmacokinetic data are thus important and needed for the potential development of these

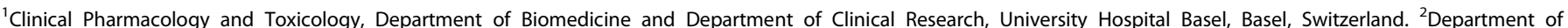

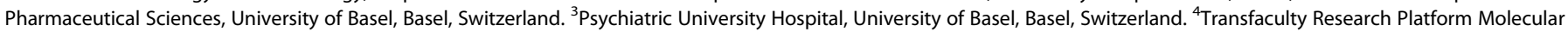
and Cognitive Neuroscience, University of Basel, Basel, Switzerland. ${ }^{{ }}$email: matthias.liechti@usb.ch
}

Received: 9 December 2021 Revised: 31 January 2022 Accepted: 8 February 2022

Published online: 25 February 2022 
substances into medications. Both LSD and psilocybin have previously been shown to induce dose-dependent cardiovascular stimulation and influence endocrine functions [11, 15, 21-23]. However, potential differences between these two substances are unexplored. Therefore, we assessed blood pressure, heart rate, body temperature, and endocrine effects, including plasma levels of cortisol, prolactin (PRL), and oxytocin. In addition, we assessed plasma levels of brain-derived neurotrophic factor (BDNF), which is considered a possible marker of neurogenesis [24] and has been shown to increase following psychedelic administration [7, 25-27].

\section{METHODS AND MATERIALS Study design}

The study used a double-blind, placebo-controlled, crossover design with five experimental test sessions to investigate responses to (i) placebo, (ii) $100 \mu \mathrm{g} \mathrm{LSD}$, (iii) $200 \mu \mathrm{g} \mathrm{LSD}$, (iv) $15 \mathrm{mg}$ psilocybin, and (v) $30 \mathrm{mg}$ psilocybin. The order of administration was random and counterbalanced. The washout periods between sessions were at least 10 days. The study was conducted in accordance with the Declaration of Helsinki and International Conference on Harmonization Guidelines in Good Clinical Practice and approved by the Ethics Committee of Northwest Switzerland (EKNZ) and Swiss Federal Office for Public Health. The study was registered at ClinicalTrials.gov (NCT03604744).

\section{Participants}

Twenty-eight healthy participants ( 14 men and 14 women; mean age \pm SD: $35 \pm 9.4$ years; range: $25-52$ years) were recruited by word of mouth or from a pool of volunteers who had contacted our research group because they were interested in participating in a clinical trial that investigated psychedelics. All of the subjects provided written informed consent and were paid for their participation. Exclusion criteria were age $<25$ years or $>65$ years, pregnancy (urine pregnancy test at screening and before each test session), personal or family (first-degree relative) history of major psychiatric disorders (assessed by the Semi-structured Clinical Interview for Diagnostic and Statistical Manual of Mental Disorders, 4th edition, Axis I disorders by a trained psychiatrist), the use of medications that may interfere with the study medications (e.g., antidepressants, antipsychotics, and sedatives), chronic or acute physical illness (e.g., abnormal physical exam, electrocardiogram, or hematological and chemical blood analyses), tobacco smoking ( $>10$ cigarettes/day), lifetime prevalence of illicit drug use $>10$ times (except for $\Delta^{9}$-tetrahydrocannabinol), illicit drug use within the last 2 months, and illicit drug use during the study period (determined by urine drug tests). The participants were asked to consume no more than 10 standard alcoholic drinks/week and have no more than one drink on the day before the test sessions. Fourteen participants had previously used a psychedelic, including LSD (11 participants, 1-5 times), psilocybin (six participants, 1-3 times), and O-methyl-bufotenin (5-MeO-DMT; one participant, two times), 12 participants had used methylenedioxymethamphetamine (MDMA; 1-5 times), 13 participants had previously used a stimulant, including methylphenidate (two participants, once), amphetamine (nine participants, 1-5 times), and cocaine (six participants, 1-2 times), one participant had used 4-bromo-2,5-dimethoxyphenethylamine (2C-B; once), and one participant had used ketamine (once). Ten participants had never used any illicit drugs with the exception of cannabis.

\section{Study drugs}

LSD base (>99\% purity; Lipomed AG, Arlesheim, Switzerland) was administered as an oral solution that was produced according to good manufacturing practice in units that contained $100 \mu \mathrm{g} \mathrm{LSD} \mathrm{in} 1 \mathrm{ml}$ of $96 \%$ ethanol [19]. The exact analytically confirmed LSD base content (mean \pm SD) was $84.5 \pm 0.98 \mu \mathrm{g}$ ( $n=10$ samples). Stability of the formulation for longer than the study period was documented in an identically produced previous batch [19]. Placebo consisted of identical units that were filled with ethanol only. Psilocybin (99.7\% purity; ReseaChem GmbH, Burgdorf, Switzerland) was administered in opaque capsules that contained a $5 \mathrm{mg}$ dose of psilocybin dihydrate and an exact analytically confirmed actual psilocybin content of $4.61 \pm 0.09 \mathrm{mg}$ (mean $\pm S D, n=10$ samples). The stability of both LSD and psilocybin products was confirmed after the study ended. Placebo consisted of identical opaque capsules that were filled with mannitol. A double-dummy method was used. The subjects received six capsules and two solutions in each session: (i) six placebo capsules and placebo/placebo solutions, (ii) six placebo capsules and 100 $\mu \mathrm{g} \mathrm{LSD/placebo} \mathrm{solutions,} \mathrm{(iii)} \mathrm{six} \mathrm{placebo} \mathrm{capsules} \mathrm{and} 100 \mu \mathrm{g} \mathrm{LSD/100 \mu g}$ LSD solutions, (iv) three $5 \mathrm{mg}$ psilocybin and three placebo capsules and placebo/placebo solutions, and (v) six $5 \mathrm{mg}$ psilocybin capsules and placebo/placebo solutions. At the end of each session and at the end of the study, the participants were asked to retrospectively guess their treatment assignment.

\section{Study procedures}

The study included a screening visit, five $25 \mathrm{~h}$ test sessions, and an end-ofstudy visit. Test days were separated by at least 10 days. The sessions were conducted in a calm hospital room. Only one research subject and one investigator were present during each test session. The test sessions began at 8:00 AM. A urine sample was taken to verify abstinence from drugs of abuse, and a urine pregnancy test was performed in women. The subjects then underwent baseline measurements. LSD, psilocybin, or placebo was administered at 9:00 AM. The outcome measures were repeatedly assessed for $24 \mathrm{~h}$. Standardized lunches and dinners were served at 1:30 PM and 6:00 PM, respectively. The subjects were never alone during the acute effect phase, and the investigator was in a room next to the subject for up to $24 \mathrm{~h}$. The subjects were sent home the next day at $\sim 9: 15 \mathrm{AM}$.

\section{Subjective drug effects and effect durations}

Subjective effects were assessed repeatedly using visual analog scales (VASs) $[21,28] 1 \mathrm{~h}$ before and $0,0.25,0.5,0.75,1,1.5,2,2.5,3,3.5,4,5,6,7$, $8,9,10,11,12,14,16$, and $24 \mathrm{~h}$ after drug administration. The Adjective Mood Rating Scale (AMRS) [29] was used $1 \mathrm{~h}$ before and 3, 6, 9, 12, and 24 $\mathrm{h}$ after drug administration. The 5 Dimensions of Altered States of Consciousness (5D-ASC) scale $[30,31]$ was used as the primary outcome measure and was administered $24 \mathrm{~h}$ after drug administration to retrospectively rate peak drug effects. Mystical experiences were assessed $24 \mathrm{~h}$ after drug administration using the States of Consciousness Questionnaire [32, 33] that includes the 43-item Mystical Effects Questionnaire (MEQ43) [32], 30-item Mystical Effects Questionnaire (MEQ30) [34], and subscales for "aesthetic experience" and negative "nadir" effects. Subjective effect measurements are described in detail in the Supplementary Methods online.

The time to onset, time to maximal effect, time to offset, and effect duration were assessed using the classic pharmacokineticpharmacodynamic (PK-PD) link module in Phoenix WinNonlin 8.3 (Certara, Princeton, NJ, USA) using the "any drug effect" VAS effect-time plots and an onset/offset threshold of $10 \%$ of the maximum individual response as described previously in detail $[7,19]$.

\section{Autonomic and adverse effects}

Blood pressure, heart rate, and tympanic body temperature were repeatedly measured at baseline and $0,0.25,0.5,0.75,1,1.5,2,2.5,3$, $3.5,4,5,6,7,8,9,10,11,12,14,16$, and $24 \mathrm{~h}$ after drug administration [35]. Pupil size was assessed at baseline and 1, 2.5, 4, 7, 11, and $24 \mathrm{~h}$ after drug administration [21]. Adverse effects were assessed $1 \mathrm{~h}$ before and 12 and $24 \mathrm{~h}$ after drug administration using the List of Complaints (LC) [36].

\section{Endocrine effects and BDNF}

Plasma concentrations of cortisol, PRL, oxytocin, and BDNF were determined as previously described [7, 21, 22, 28]. Cortisol, PRL, and oxytocin were measured before and $2.5 \mathrm{~h}$ after drug administration. Plasma BDNF levels were measured at baseline and 4,6 , and $12 \mathrm{~h}$ after drug administration.

\section{Plasma LSD and psilocin concentrations}

Blood was collected into lithium heparin tubes. The blood samples were immediately centrifuged, and the plasma was subsequently stored at $-80^{\circ}$ $C$ until analysis. Plasma concentrations of LSD were determined by ultrahigh-performance liquid chromatography tandem mass spectrometry with a lower limit of quantification of $10 \mathrm{pg} / \mathrm{ml}$ [19]. Plasma psilocin concentrations were analyzed using ultra-high-performance liquid chromatography tandem mass spectrometry as described previously [37]. Both methods were fully validated. 


\section{Pharmacokinetic analyses}

Pharmacokinetic parameters were estimated using non-compartmental methods as described previously [19]. Analyses were conducted using Phoenix WinNonlin 8.3 (Certara, Princeton, NJ, USA).

\section{Data analysis}

Peak $\left(E_{\max }\right.$ and/or $\left.E_{\min }\right)$ or peak change from baseline $\left(\Delta E_{\max }\right)$ values were determined for repeated measures. The values were then analyzed using repeated-measures analysis of variance (ANOVA), with drug as the withinsubjects factor, followed by the Tukey post hoc tests using Statistica 12 software (StatSoft, Tulsa, OK, USA). The criterion for significance was $p<0.05$.

\section{RESULTS}

\section{Subjective drug effects}

Alterations of mind and mystical-type effects are shown in Fig. 1 and Supplementary Fig. S1, respectively. Statistics are summarized in Supplementary Tables S1-2. Subjective effects over time on the VAS are shown in Fig. 2 and Supplementary Fig. S2. Effects on mood over time on the AMRS are shown in Supplementary Fig. S3. The corresponding peak responses and statistics are presented in Supplementary Tables S3-4. Characteristics of subjective responses derived from the PK-PD model are shown in Table 1.

LSD at doses of 100 and $200 \mu \mathrm{g}$ and the psilocybin dose of 30 $\mathrm{mg}$ produced comparable subjective effects. Specifically, $200 \mu \mathrm{g}$ LSD produced comparable positive drug effects to $100 \mu \mathrm{g} \mathrm{LSD}$, but the higher dose produced greater ego-dissolution $(p<0.05)$ and a trend toward an increase in anxiety $(p=0.054)$. The high $30 \mathrm{mg}$ psilocybin dose produced comparable maximal subjective effects to the 100 and $200 \mu \mathrm{g}$ LSD doses, with no significant differences in any of the VASs or 5D-ASC subscales. The $200 \mu \mathrm{g}$ LSD and $30 \mathrm{mg}$ psilocybin doses differed only in ratings of "ineffability" on the MEQ. Effects of the $15 \mathrm{mg}$ psilocybin dose were significantly lower than the $200 \mu \mathrm{g}$ LSD dose on the VASs "any drug effect" $(p<$ $0.001)$, "good drug effect" $(p<0.001)$, "stimulated" $(p<0.001)$, "talkative" $(p<0.01)$, "perception of time" $(p<0.001)$, and "egodissolution" ( $p<0.001)$ and 5D-ASC total scores $(p<0.001)$ and all main dimensions, including anxious ego dissolution $(p<0.001)$, oceanic boundlessness $(p<0.001)$, and visionary restructuralization $(p<0.001)$. The $15 \mathrm{mg}$ psilocybin dose produced clearly lower effects than the $30 \mathrm{mg}$ psilocybin and both LSD doses.

On the AMRS, effects of 100 and $200 \mu \mathrm{g}$ LSD were generally nominally higher than 15 and $30 \mathrm{mg}$ psilocybin, and both LSD doses significantly increased "emotional excitation" compared with psilocybin.

Both LSD doses had significantly longer effect durations and an earlier onset of effects compared with both doses of psilocybin (Table 1).

There was no difference in the subjective effects of LSD and psilocybin between male and female participants.

\section{Autonomic and adverse effects}

Autonomic effects over time and respective peak effects are shown in Fig. 3 and Supplementary Table S5, respectively. Frequently reported adverse effects, systematically assessed by the LC, are presented in Supplementary Table S6. Both LSD and psilocybin significantly increased diastolic and systolic blood pressure, body temperature, and pupil size compared with placebo. LSD increased blood pressure and body temperature only moderately, whereas $30 \mathrm{mg}$ psilocybin produced significantly greater increases in blood pressure and body temperature compared with LSD and $15 \mathrm{mg}$ psilocybin. In contrast, both LSD doses produced a greater increase in heart rate compared with both psilocybin doses and placebo. Psilocybin at a dose of $30 \mathrm{mg}$ but not $15 \mathrm{mg}$ moderately increased heart rate compared with placebo.
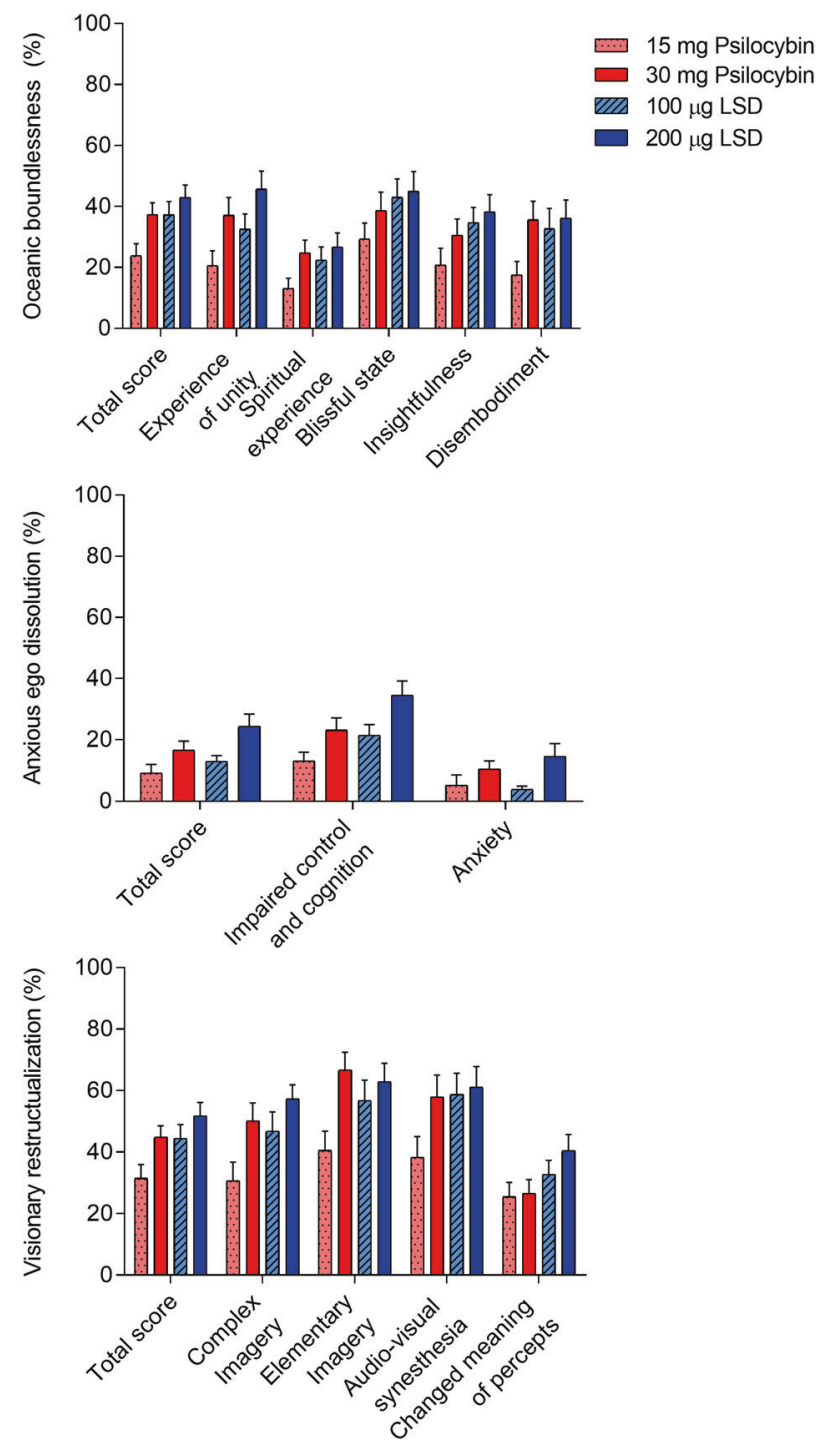

Fig. 1 Acute alterations of mind on the 5 Dimensions of Altered States of Consciousness (5D-ASC) scale. Psilocybin at $30 \mathrm{mg}$ produced alterations of mind that were nominally similar to 100 $\mu \mathrm{g}$ LSD and not significantly different from either 100 or $200 \mu \mathrm{g}$ LSD. LSD at 100 and $200 \mu \mathrm{g}$ significantly differed only in the "Anxious Ego Dissolution" total score and the "impaired control and cognition" and "anxiety" subscales. Effects of the $15 \mathrm{mg}$ psilocybin dose were clearly lower than 100 and $200 \mu \mathrm{g}$ LSD and $30 \mathrm{mg}$ psilocybin on most subscales. Placebo scores were too low for visualization. The data are expressed as the mean \pm SEM percentage of maximally possible scale scores in 28 subjects. Statistics are shown in Supplementary Table S1.

Both LSD and psilocybin increased pupil size at both doses (Supplementary Fig. S4 and Supplementary Table S5). Furthermore, $200 \mu \mathrm{g}$ LSD and $30 \mathrm{mg}$ psilocybin significantly impaired normal light-induced pupil constriction compared with placebo.

LSD and psilocybin at both doses increased the total acute (0-12 h) adverse effect score on the LC compared with placebo. Subacute (12-24h) adverse effect scores were significantly increased by the high doses ( $200 \mu \mathrm{g}$ LSD and $30 \mathrm{mg}$ psilocybin) compared with placebo. There was no difference in the autonomic and adverse effects of LSD and psilocybin between male and female participants.

LSD and psilocybin also resulted in additional adverse events in the evening on the treatment day or within $48 \mathrm{~h}$ after treatment. 

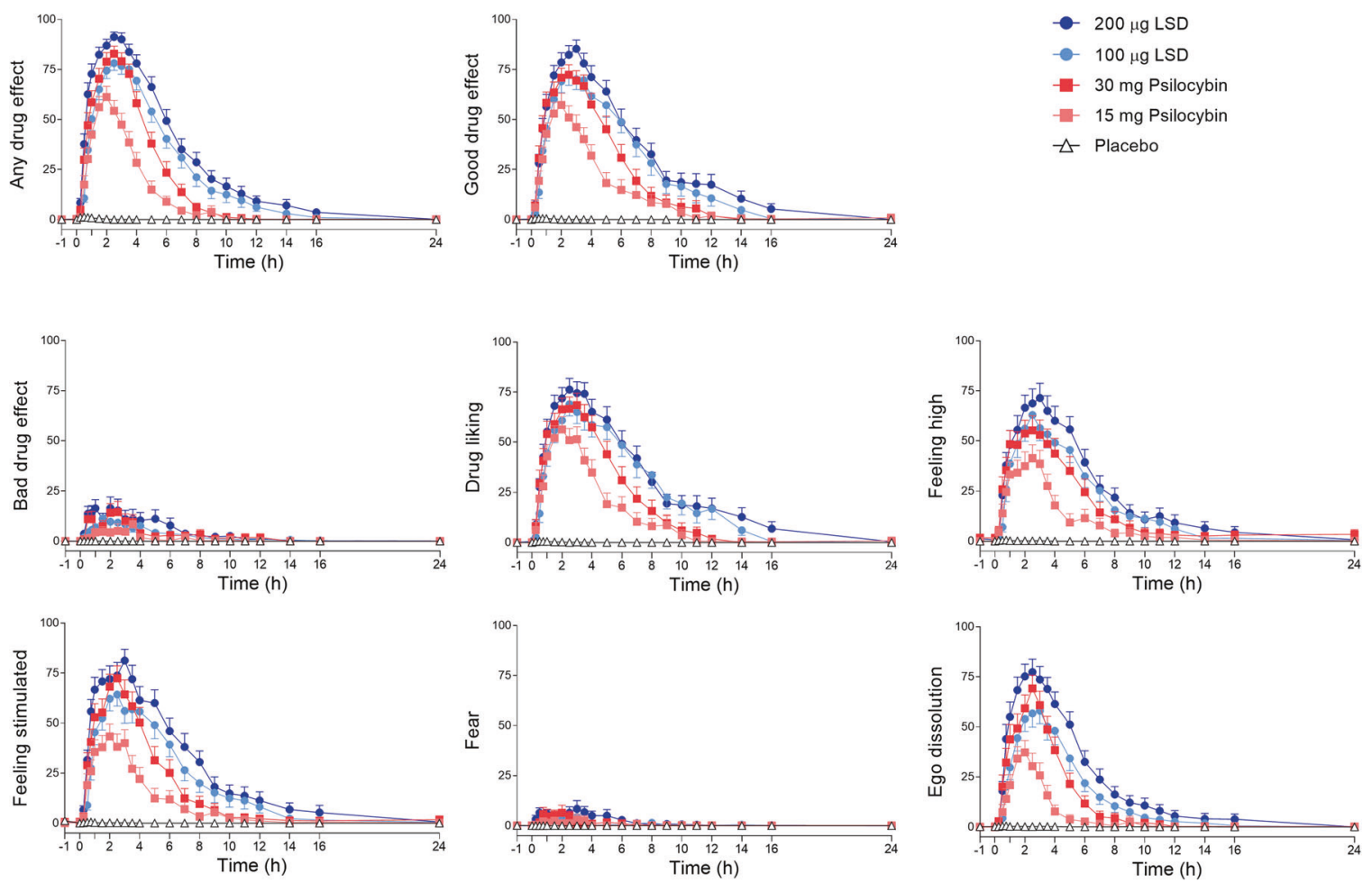

Fig. 2 Acute subjective effects induced by lysergic acid diethylamide (LSD) and psilocybin over time on the Visual Analog Scale (VAS). LSD $(100$ or $200 \mu \mathrm{g}$ ), psilocybin $(15$ or $30 \mathrm{mg}$ ), or placebo was administered at $t=0 \mathrm{~h}$. Generally, the LSD doses of $100 \mu \mathrm{g}$ and $200 \mu \mathrm{g}$ and psilocybin dose of $30 \mathrm{mg}$ produced comparable subjective effects on the VASs "any drug effect," "good drug effect," "bad drug effect," "drug liking," "feeling high," "feeling stimulated," and "fear." Only the VAS "ego dissolution" showed a significant difference between 100 and 200 ug LSD. The high $30 \mathrm{mg}$ psilocybin dose produced maximal subjective effects that were comparable to 100 and $200 \mu \mathrm{g}$ LSD, with no significant differences on any of the VASs. The $30 \mathrm{mg}$ psilocybin dose produced significantly greater peak responses than the $15 \mathrm{mg}$ psilocybin dose on the VAS "any drug effect," "good drug effect," "feeling stimulated," and "ego dissolution." The data are expressed as the mean \pm SEM percentage of maximally possible scale scores in 28 subjects. The corresponding maximal responses and statistics are shown in Supplementary Table S3.

Table 1. Characteristics of the subjective response to different doses of LSD and psilocybin.

\begin{tabular}{|c|c|c|c|c|c|c|}
\hline Effect & 15 mg Psilocybin & 30 mg Psilocybin & $100 \mu \mathrm{g}$ LSD & $200 \mu \mathrm{g}$ LSD & $F_{3,81}$ & $\boldsymbol{P}=$ \\
\hline Time to onset (h) & $0.8 \pm 0.3(0.3-1.5)$ & $0.8 \pm 0.4(0.1-1.8)$ & $0.6 \pm 0.2^{\#}(0.3-1.0)$ & 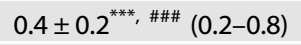 & 12.5 & $<0.001$ \\
\hline Time to offset (h) & $6.4 \pm 2.1(3.7-11)$ & $7.3 \pm 2.3(4.2-12)$ & 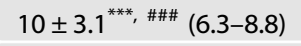 & 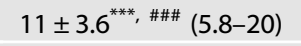 & 31.2 & $<0.001$ \\
\hline Time to maximal effect $(h)$ & $2.1 \pm 0.5(1.5-3.4)$ & $2.3 \pm 0.8(0.5-3.3)$ & $2.5 \pm 0.5^{* *}(1.8-2.4)$ & $2.2 \pm 0.6(1.2-3.9)$ & 3.8 & $<0.05$ \\
\hline Effect duration (h) & $5.6 \pm 2.2(2.5-10)$ & $6.5 \pm 2.4(3.7-12)$ & 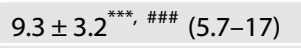 & 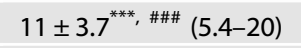 & 34.4 & $<0.001$ \\
\hline Maximal effect (\%) & $58 \pm 25(13-98)$ & $80 \pm 18 * * *(43-100)$ & $77 \pm 18^{* * *}(33-100)$ & $87 \pm 13^{* * *}(46-100)$ & 20.0 & $<0.001$ \\
\hline
\end{tabular}

Parameters are for "any drug effects" as predicted by the PK-PD link model. The threshold to determine times to onset and offset was set individually at $10 \%$ of the individual maximal response. Values are mean \pm SD (range).

${ }^{* *} P<0.01,{ }^{* * *} P<0.001$ compared with $15 \mathrm{mg}$ of psilocybin; ${ }^{\#} P<0.05,{ }^{\# \# \#} P<0.001$ compared with $30 \mathrm{mg}$ of psilocybin.

Adverse aftereffects included headaches (four subjects after psilocybin and three after LSD), migraine attack (one subject after LSD), nosebleeds (one subject after psilocybin), low mood (two subjects after psilocybin and two subjects after LSD), nausea (two subjects after psilocybin and one subject after LSD), nightmares (one subject after psilocybin), restlessness (one subject after psilocybin and one subject after LSD), vivid dreams (one subject after LSD), insomnia (one subject after psilocybin), and involuntary movement of the lower extremities (one subject after LSD). Nine flashback episodes occurred in five subjects (one to 20 times within $72 \mathrm{~h}$ after substance administration; five episodes after LSD administration and four episodes after psilocybin administration). Altogether, the type and number of adverse events after acute psilocybin and LSD administration were comparable. No severe adverse events were observed.

\section{Endocrine effects and BDNF}

Effects of LSD and psilocybin on plasma levels of cortisol, PRL, oxytocin, and BDNF are shown in Supplementary Fig. S5 and Supplementary Table S5. Both LSD and psilocybin significantly increased plasma cortisol, PRL, and oxytocin levels. Neither LSD nor psilocybin significantly elevated plasma BDNF levels.

\section{Plasma drug concentrations}

The concentration-time curves for LSD and psilocin are shown in Supplementary Fig. S6. Supplementary Table S7 shows the pharmacokinetic parameters. The geometric mean maximum $\left(C_{\text {max }}\right)$ values (range) for 100 and $200 \mu \mathrm{g}$ LSD were $1.9(1.1-3.3)$ $\mathrm{ng} / \mathrm{ml}$ and $3.4(1.6-5.8) \mathrm{ng} / \mathrm{ml}$, respectively. The $T_{\max }$ values were $1.6(1.75-3.0) h$ and $1.6(1.3-3.5) h$, respectively. Elimination halflives $\left(t_{1 / 2}\right)$ were $4.3(2.5-6.0) h$ and $4.0(2.3-7.1) h$, respectively. The 


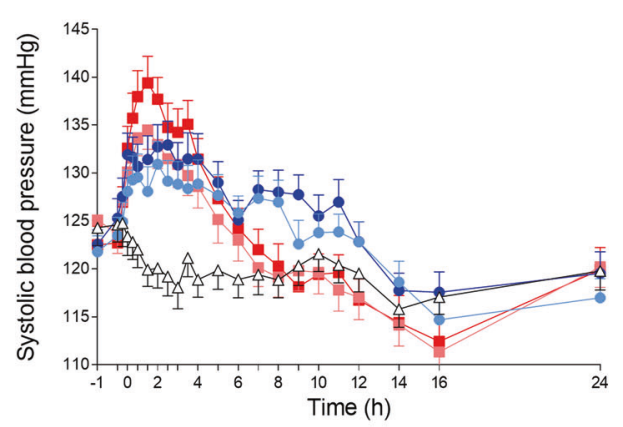
$\triangle$ Placebo
- $100 \mu \mathrm{g}$ LSD
- $200 \mu \mathrm{g}$ LSD
- $15 \mathrm{mg}$ Psilocybin
- 30 mg Psilocybin
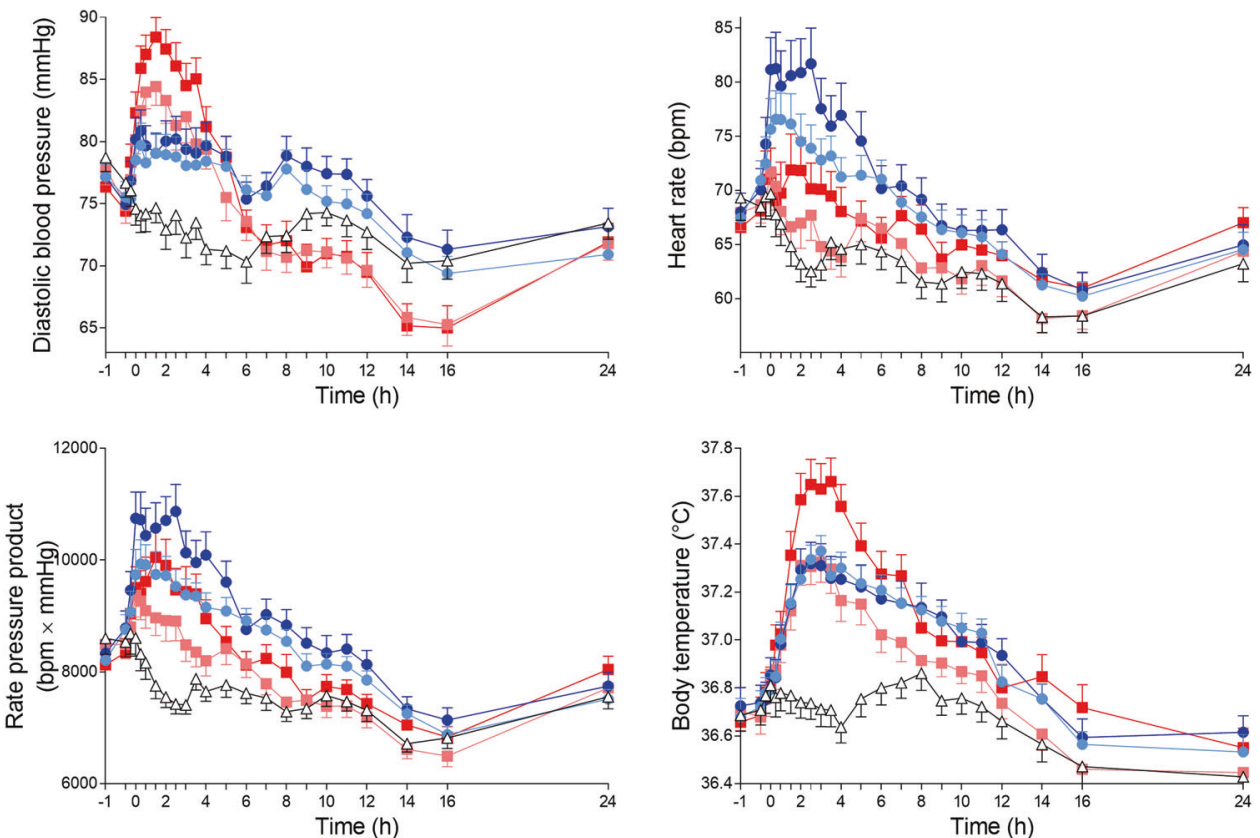

Fig. 3 Acute autonomic effects of lysergic acid diethylamide (LSD) and psilocybin over time. The 100 and $200 \mu \mathrm{g}$ doses of lysergic acid diethylamide (LSD) only moderately increased blood pressure compared with placebo, whereas 15 and 30 mg psilocybin induced more pronounced increases in blood pressure. The 100 and $200 \mu \mathrm{g}$ doses of LSD markedly increased heart rate, whereas only the higher $30 \mathrm{mg}$ dose of psilocybin induced a moderate increase in heart rate compared with placebo. Both the 100 and $200 \mu \mathrm{g}$ LSD doses and the $15 \mathrm{mg}$ psilocybin dose increased body temperature moderately and similarly, whereas $30 \mathrm{mg}$ psilocybin induced a more pronounced increase in body temperature compared with all other conditions. LSD $(100$ or $200 \mu \mathrm{g})$, psilocybin $(15 \mathrm{or} 30 \mathrm{mg})$, or placebo was administered at $t=0 \mathrm{~h}$. The data are expressed as the mean \pm SEM in 28 subjects. Maximal effects and statistics are shown in Supplementary Table S5.

$\mathrm{C}_{\max }$ values for 15 and $30 \mathrm{mg}$ psilocin were $13(7-21) \mathrm{ng} / \mathrm{ml}$ and 25 (14-41) $\mathrm{ng} / \mathrm{ml}$, respectively. The corresponding $T_{\max }$ values were $2.3(0.8-3.5) \mathrm{h}$ and $2.5(0.77-4.0) \mathrm{h}$, respectively. Elimination halflives $\left(t_{1 / 2}\right)$ were $2.4(1.7-3.1) h$ and $2.7(1.9-3.4) h$, respectively. Both LSD and psilocybin showed linear pharmacokinetics. Body weight had no influence on the pharmacokinetics of LSD or psilocybin.

\section{Blinding}

Data on the participants' retrospective identification of the substance and dose are shown in Supplementary Table S8. Overall, no clear distinction between LSD and psilocybin could be made after the sessions, nor at the end of the study. The $15 \mathrm{mg}$ dose of psilocybin was correctly identified in $64 \%$ of the sessions after the session and mistaken for $100 \mu \mathrm{g}$ LSD in $29 \%$ of the sessions. When asked after the session, the $30 \mathrm{mg}$ psilocybin dose was correctly identified in $57 \%$ of the sessions and mistaken for $15 \mathrm{mg}$ psilocybin in $29 \%$ of the sessions. The $100 \mu \mathrm{g}$ LSD dose was correctly identified in $57 \%$ of the sessions and mistaken for $200 \mu \mathrm{g}$ LSD in $21 \%$ of the sessions. The $200 \mu \mathrm{g}$ LSD dose was correctly identified in $61 \%$ of the sessions and mistaken as $100 \mu \mathrm{g}$ LSD and $30 \mathrm{mg}$ psilocybin in $18 \%$ of the sessions for both. When asked at the end of the study, $15 \mathrm{mg}$ psilocybin was mostly mistaken for 30 mg psilocybin, $30 \mathrm{mg}$ psilocybin was mostly mistaken for $100 \mu \mathrm{g}$ LSD, $100 \mu \mathrm{g}$ LSD was mostly mistaken for $15 \mathrm{mg}$ psilocybin, and $200 \mu \mathrm{g}$ LSD was mostly mistaken for $100 \mu \mathrm{g}$ LSD. Placebo could be distinguished well from active substance and correctly identified in $96 \%$ of the sessions.

\section{DISCUSSION}

The present study investigated and directly compared acute effects of LSD and psilocybin using well-defined doses in healthy participants. Previous recent studies only investigated either LSD or psilocybin alone. The present study was the first modern study that compared both substances within the same study using a within-subjects design. We used LSD and psilocybin doses that covered the range of therapeutically used doses and were expected to induce comparable subjective effects as previously reported in similar Phase 1 studies of either LSD or psilocybin $[11,21,33]$. The present study was also the first to describe acute effects and particularly the pharmacokinetics of fixed doses of psilocybin, thus complementing our recent study using a fixed dose of $25 \mathrm{mg}$ psilocybin in healthy subjects [38]. In contrast, several previous studies of psilocybin in healthy participants used body weight-adjusted dosing approaches [11, 13-15, 39, 40]. Fixed 
dosing is likely to be used in therapeutic settings $[2,41]$. The body weight adjustment of the psilocybin dose did not alter subjective effects and had no advantages [42], and fixed dosing is more practical in large trials in patients. Both LSD and psilocybin were used in pharmaceutical formulations with defined content uniformity and stability. Furthermore, plasma concentrations of both LSD and psilocin were determined as measures of exposure to the substances.

Subjective effects that were induced by both doses of LSD and the high $30 \mathrm{mg}$ dose of psilocybin were largely comparable, whereas $15 \mathrm{mg}$ psilocybin exerted clearly weaker effects. Subjective effects of LSD in the present study were similar to previous studies that investigated either LSD or psilocybin [7, 11, 28]. For LSD, the dose-effect relationship reached a ceiling effect for good drug effects at a dose of $100 \mu \mathrm{g}$. Only ego dissolution and negative drug effects further increased at a dose of $200 \mu \mathrm{g}$ compared with $100 \mu \mathrm{g}$, which is consistent with previous studies $[7,43]$. Notably, the ceiling effect was less pronounced in the present study compared with our previous study [7]. In contrast, psilocybin showed a clear dose-effect relationship on most outcome measures at the doses used in the present study. Ratings of the high $30 \mathrm{mg}$ psilocybin dose were nominally between the 100 and $200 \mu \mathrm{g}$ doses of LSD, indicating that 30 $\mathrm{mg}$ psilocybin corresponds to $150 \mu \mathrm{g}$ LSD base, a dose that was not tested herein. This means that the doses of psilocybin that were used in the present study (30 and $15 \mathrm{mg}$ ) were lower in terms of strength compared with the two doses of LSD, and ceiling effects were likely not reached for psilocybin compared with LSD. A previous study that investigated body weightadjusted doses of psilocybin of $\sim 20 \mathrm{mg} / 70 \mathrm{~kg}, 30 \mathrm{mg} / 70 \mathrm{~kg}$, and $40 \mathrm{mg} / 70 \mathrm{~kg}$ found no difference in positive mood scale ratings on the MEQ30 [14], indicating that a ceiling effect for good drug effects for psilocybin, similar to LSD, might be reached at doses above $20 \mathrm{mg}$. The only subscale on which $200 \mu \mathrm{g}$ LSD was significantly different from $30 \mathrm{mg}$ psilocybin was "ineffability" on the MEQ30 and MEQ43. Ineffability largely describes the ability to express an experience in words. Because $200 \mu \mathrm{g}$ LSD was nominally more effective than $30 \mathrm{mg}$ psilocybin and because $100 \mu \mathrm{g}$ LSD showed a nearly significant lower effect on this scale compared with $200 \mu \mathrm{g}$ LSD, we suggest that this is a dose effect rather than a substance-specific effect. In addition, no sex differences were found, consistent with previous reports $[7,28,43]$

Both LSD and psilocybin had dose-dependent effect durations, with higher doses producing longer effects. However, the effects of LSD were also clearly and significantly longer than the effects of psilocybin. The differences in the duration of action can be fully explained by differences in the pharmacokinetics of LSD and psilocin. The elimination half-life values of LSD and psilocin were an average of $\sim 4 \mathrm{~h}$ and $2.5 \mathrm{~h}$, respectively. These values are consistent with previous studies [7, 13, 19,37], although a slightly shorter half-life of $2 \mathrm{~h}$ has also been described for psilocybin $[38,44]$. Body weight had no influence on LSD or psilocin plasma concentrations, as described previously [7, 38, 45]. The faster time of onset for LSD can be explained by the liquid formulation compared with the capsules that were used for psilocybin and cannot be attributed to the substance.

LSD and psilocybin both produced significant autonomic stimulant effects as observed previously [7, 11, 40, 43]. The cardiostimulant responses were present at both doses, with a trend toward greater responses at the higher doses. Interestingly, psilocybin produced stronger elevations of arterial blood pressure, whereas LSD produced stronger elevations of heart rate. When combining elevations of heart rate and blood pressure into the rate-pressure product, the high dose of psilocybin $(30 \mathrm{mg})$ and both doses of LSD $(100$ and $200 \mu \mathrm{g})$ exerted overall similar cardiovascular stimulation, whereas the $15 \mathrm{mg}$ dose of psilocybin exerted overall weaker effects. Psilocybin increased body temperature more than LSD. Psilocybin also produced greater impairments in pupil contraction compared with LSD. This lower pupillary contraction in response to a light stimulus has also been observed with MDMA compared with LSD [28] and may represent a similarity of psilocybin and MDMA. This similarity may be explained by a common action of psilocybin and MDMA on the serotonin transporter [10]. Overall, however, these autonomic effects were moderate and transient and thus not a safety concern. We also assessed acute and subacute adverse effects and spontaneously reported adverse events between test days. LSD and psilocybin produced comparable acute adverse effects, but the high doses ( $30 \mathrm{mg}$ psilocybin and $200 \mu \mathrm{g}$ LSD) produced more subacute adverse effects, indicating that higher doses are associated with longer-lasting and more unpleasant effects. The number and type of systematically assessed and spontaneous reported adverse effects are comparable to those reported in a larger pooled analysis of the safety of LSD in healthy subjects [43].

The true content of the LSD formulation in the present study was $11-14 \%$ lower than in previous studies by our group that used the same dose and formulation [7, 19, 28]. This lower LSD content and larger study group size might partly explain the trend toward greater effect differences between the 100 and $200 \mu \mathrm{g}$ LSD doses compared with a previous dose-finding study in 16 subjects [7]. The two doses of psilocybin ( 15 and $30 \mathrm{mg}$ ) that were selected for this study were lower in terms of acute effect strength compared with the 100 and $200 \mu \mathrm{g}$ doses of LSD. We also recently evaluated the acute effects of $25 \mathrm{mg}$ psilocybin. Altogether, these results suggest that $20 \mathrm{mg}$ psilocybin is equivalent to $100 \mu \mathrm{g}$ LSD, and 30 $\mathrm{mg}$ psilocybin is equivalent to $150 \mu \mathrm{g} L \mathrm{SD}$, a consistency that was also noted elsewhere [46]. Thus, the dose equivalence of LSD to psilocybin is 1:200. This result may be helpful for dose finding in future studies and facilitate interpretations of future clinical results that are obtained with either substance.

The present study was well blinded. The only condition that was identified by the subjects with high certainty was placebo. Furthermore, the high dose of LSD $(200 \mu \mathrm{g})$ was almost never mistaken for the low dose of psilocybin $(15 \mathrm{mg})$. Generally, both the low and high doses were more likely to be confused with each other rather than the high dose being exclusively mistaken for the low dose. Interestingly, this was still the case at the end of the study, despite the clear differences in effect durations between LSD and psilocybin that could be expected to unmask the blinding between substances. These findings further support the view that alterations of states of consciousness that are induced by LSD and psilocybin are more likely dose-dependent rather than substancedependent and that the differences in their pharmacological profiles [10] do not relevantly influence subjectively experienced effects. Studies in rats indicated a later, more negative, temporal phase with involvement of $D_{2}$ and $D_{4}$ receptors for LSD, but not for psilocybin $[47,48]$. The finding of no difference in the quality of subjective effects of LSD and psilocybin also confirms that both classic psychedelics produce their effects via shared agonistic effects on $5-\mathrm{HT}_{2 \mathrm{~A}}$ receptors. The subjective effects of both substances can robustly be blocked by $5-\mathrm{HT}_{2 \mathrm{~A}}$ receptor antagonist administration in humans [7-9].

In the present study, neither LSD nor psilocybin altered plasma BDNF concentrations compared with placebo. Previous studies reported that $200 \mu \mathrm{g}$ LSD but not $100 \mu \mathrm{g}$ LSD increased BDNF levels $[7,28]$. In addition, LSD and psilocybin both increased PRL and cortisol levels, which are markers of serotonergic activity [49]. Furthermore, the present study was the first to document increases in circulating oxytocin after psilocybin administration as previously shown for LSD $[21,22]$ and MDMA $[28,50]$.

The present study has strengths. Two well-characterized doses of LSD and psilocybin were used within-subjects and compared with placebo under double-blind conditions in a laboratory setting. We included equal numbers of male and female participants and used internationally established psychometric 
outcome measures. Plasma LSD and psilocin concentrations were determined up to $24 \mathrm{~h}$ for all conditions. Notwithstanding these strengths, the present study also has limitations. The study used a highly controlled setting and included only healthy subjects. Thus, subjects in different environments and patients with psychiatric disorders may respond differently to either LSD or psilocybin.

\section{CONCLUSION}

We characterized the effects of LSD and psilocybin at two different doses to support dose finding for research and psychedelicassisted therapy. The $20 \mathrm{mg}$ dose of psilocybin is likely equivalent to the $100 \mu \mathrm{g}$ dose of LSD base. We found no evidence of qualitative differences in altered states of consciousness that were induced by either LSD or psilocybin, except that the duration of action was shorter for psilocybin.

\section{REFERENCES}

1. Krebs TS, Johansen PO. Over 30 million psychedelic users in the United States. F1000Research. 2013;2:98.

2. Carhart-Harris R, Giribaldi B, Watts R, Baker-Jones M, Murphy-Beiner A, Murphy R, et al. Trial of psilocybin versus escitalopram for depression. $\mathrm{N}$ Engl J Med. 2021;384:1402-11.

3. Griffiths RR, Johnson MW, Carducci MA, Umbricht A, Richards WA, Richards BD, et al. Psilocybin produces substantial and sustained decreases in depression and anxiety in patients with life-threatening cancer: a randomized double-blind trial. J Psychopharmacol. 2016;30:1181-97.

4. Davis AK, Barrett FS, May DG, Cosimano MP, Sepeda ND, Johnson MW, et al. Effects of psilocybin-assisted therapy on major depressive disorder: a randomized clinical trial. JAMA Psychiatry. 2021;78:481-9.

5. Gasser P, Kirchner K, Passie T. LSD-assisted psychotherapy for anxiety associated with a life-threatening disease: a qualitative study of acute and sustained subjective effects. J Psychopharmacol. 2015;29:57-68.

6. Schindler EAD, Sewell RA, Gottschalk CH, Luddy C, Flynn LT, Lindsey $H$, et al. Exploratory Controlled Study of the Migraine-Suppressing Effects of Psilocybin. Neurotherapeutics 2020;18:534-43.

7. Holze F, Vizeli P, Ley L, Muller F, Dolder P, Stocker M, et al. Acute dose-dependent effects of lysergic acid diethylamide in a double-blind placebo-controlled study in healthy subjects. Neuropsychopharmacology 2021;46:537-44.

8. Vollenweider FX, Vollenweider-Scherpenhuyzen MF, Babler A, Vogel H, Hell D. Psilocybin induces schizophrenia-like psychosis in humans via a serotonin-2 agonist action. Neuroreport 1998;9:3897-902.

9. Preller $\mathrm{KH}$, Herdener M, Pokorny T, Planzer A, Kraehenmann R, Stämpfli $P$, et al. The fabric of meaning and subjective effects in LSD-induced states depend on serotonin 2A receptor activation. Curr Biol. 2017;27:451-57.

10. Rickli A, Moning OD, Hoener MC, Liechti ME. Receptor interaction profiles of novel psychoactive tryptamines compared with classic hallucinogens. Eur Neuropsychopharmacol. 2016;26:1327-37.

11. Carbonaro TM, Johnson MW, Hurwitz E, Griffiths RR. Double-blind comparison of the two hallucinogens psilocybin and dextromethorphan: similarities and differences in subjective experiences. Psychopharmacology. 2018;235:521-34.

12. Liechti ME. Modern clinical research on LSD. Neuropsychopharmacology 2017;42:2114-27.

13. Brown RT, Nicholas CR, Cozzi NV, Gassman MC, Cooper KM, Muller D, et al. Pharmacokinetics of escalating doses of oral psilocybin in healthy adults. Clin Pharmacokinetics. 2017;56:1543-54.

14. Nicholas CR, Henriquez KM, Gassman MC, Cooper KM, Muller D, Hetzel S, et al. High dose psilocybin is associated with positive subjective effects in healthy volunteers. J Psychopharmacol. 2018;32:770-8.

15. Hasler F, Grimberg U, Benz MA, Huber T, Vollenweider FX. Acute psychological and physiological effects of psilocybin in healthy humans: a double-blind, placebo-controlled dose-effect study. Psychopharmacology 2004;172:145-56.

16. Roseman L, Nutt DJ, Carhart-Harris RL. Quality of acute psychedelic experience predicts therapeutic efficacy of psilocybin for treatment-resistant depression. Front Pharmacol. 2017;8:974.

17. Ross S, Bossis A, Guss J, Agin-Liebes G, Malone T, Cohen B, et al. Rapid and sustained symptom reduction following psilocybin treatment for anxiety and depression in patients with life-threatening cancer: a randomized controlled trial. J Psychopharmacol. 2016;30:1165-80.

18. Garcia-Romeu A, Griffiths RR, Johnson MW. Psilocybin-occasioned mystical experiences in the treatment of tobacco addiction. Curr Drug Abus Rev. 2015;7:157-64.
19. Holze F, Duthaler U, Vizeli P, Muller F, Borgwardt S, Liechti ME. Pharmacokinetics and subjective effects of a novel oral LSD formulation in healthy subjects. $\mathrm{Br} J$ Clin Pharmacol. 2019;85:1474-83.

20. Madsen MK, Fisher PM, Burmester D, Dyssegaard A, Stenbaek DS, Kristiansen S, et al. Psychedelic effects of psilocybin correlate with serotonin $2 A$ receptor occupancy and plasma psilocin levels. Neuropsychopharmacology 2019;44:1328-34.

21. Schmid Y, Enzler F, Gasser P, Grouzmann E, Preller KH, Vollenweider FX, et al. Acute effects of lysergic acid diethylamide in healthy subjects. Biol Psychiatry 2015;78:544-53.

22. Holze F, Avedisian I, Varghese N, Eckert A, Liechti ME. Role of the 5-HT $2 \mathrm{~A}$ Receptor in acute effects of LSD on empathy and circulating oxytocin. Front Pharm. 2021;12:711255.

23. Gouzoulis-Mayfrank E, Thelen B, Habermeyer E, Kunert HJ, Kovar KA, Lindenblatt $H$, et al. Psychopathological, neuroendocrine and autonomic effects of 3,4methylenedioxyethylamphetamine (MDE), psilocybin and d-methamphetamine in healthy volunteers: results of an experimental double blind placebo controlled study. Psychopharmacology 1999;142:41-50.

24. Haile CN, Murrough JW, losifescu DV, Chang LC, Al Jurdi RK, Foulkes A, et al. Plasma brain derived neurotrophic factor (BDNF) and response to ketamine in treatment-resistant depression. Int J Neuropsychopharmacol. 2014;17:331-6.

25. Ly C, Greb AC, Cameron LP, Wong JM, Barragan EV, Wilson PC, et al. Psychedelics promote structural and functional neural plasticity. Cell Rep. 2018;23:3170-82.

26. de Almeida RN, Galvao ACM, da Silva FS, Silva E, Palhano-Fontes F, Maia-deOliveira JP, et al. Modulation of serum brain-derived neurotrophic factor by a single dose of ayahuasca: observation from a randomized controlled trial. Front Psychol. 2019;10:1234.

27. Hutten N, Mason NL, Dolder P, Theunissen EL, Holze F, Liechti ME, et al. Low dose LSD acutely increases BDNF blood plasma levels in healthy volunteers. ACS Pharm Transl Sci. 2020;4:461-6.

28. Holze F, Vizeli P, Muller F, Ley L, Duerig R, Varghese N, et al. Distinct acute effects of LSD, MDMA, and D-amphetamine in healthy subjects. Neuropsychopharmacology 2020;45:462-71.

29. Janke W, Debus G. Die Eigenschaftswörterliste. Göttingen:Hogrefe;1978.

30. Dittrich A. The standardized psychometric assessment of altered states of consciousness (ASCs) in humans. Pharmacopsychiatry 1998;31:80-4. Suppl 2

31. Studerus E, Gamma A, Vollenweider FX. Psychometric evaluation of the altered states of consciousness rating scale (OAV). PLoS ONE. 2010;5:e12412.

32. Griffiths RR, Richards WA, McCann U, Jesse R. Psilocybin can occasion mysticaltype experiences having substantial and sustained personal meaning and spiritual significance. Psychopharmacology 2006;187:268-83. discussion 84-92

33. Liechti ME, Dolder PC, Schmid Y. Alterations in conciousness and mystical-type experiences after acute LSD in humans. Psychopharmacology 2017;234:1499-510.

34. Barrett FS, Johnson MW, Griffiths RR. Validation of the revised Mystical Experience Questionnaire in experimental sessions with psilocybin. J Psychopharmacol. 2015;29:1182-90.

35. Hysek CM, Vollenweider FX, Liechti ME. Effects of a $\beta$-blocker on the cardiovascular response to MDMA (ecstasy). Emerg Med J. 2010;27:586-9.

36. Zerssen DV. Die Beschwerden-Liste. Münchener Informationssystem. München: Psychis;1976.

37. Kolaczynska KE, Liechti ME, Duthaler U. Development and validation of an LC-MS/ MS method for the bioanalysis of psilocybin's main metabolites, psilocin and 4hydroxyindole-3-acetic acid, in human plasma. J Chromatogr B Anal Technol Biomed Life Sci. 2021;1164:122486.

38. Becker AM, Holze F, Grandinetti T, Klaiber A, Toedtli VE, Kolaczynska KE, et al. Acute effects of psilocybin after escitalopram or placebo pretreatment in a randomized, double-blind, placebo-controlled, cross-over study in healthy subjects. Clin Pharmacol Ther. 07 November 2021. https://doi.org/10.1002/cpt.2487

39. Studerus E, Kometer M, Hasler F, Vollenweider FX. Acute, subacute and long-term subjective effects of psilocybin in healthy humans: a pooled analysis of experimental studies. J Psychopharmacol. 2011;25:1434-52.

40. Griffiths RR, Johnson MW, Richards WA, Richards BD, McCann U, Jesse R. Psilocybin occasioned mystical-type experiences: immediate and persisting doserelated effects. Psychopharmacology 2011;218:649-65.

41. Carhart-Harris RL, Bolstridge M, Rucker J, Day CM, Erritzoe D, Kaelen M, et al. Psilocybin with psychological support for treatment-resistant depression: an open-label feasibility study. Lancet Psychiatry. 2016;3:619-27.

42. Garcia-Romeu A, Barrett FS, Carbonaro TM, Johnson MW, Griffiths RR. Optimal dosing for psilocybin pharmacotherapy: considering weight-adjusted and fixed dosing approaches. J Psychopharmacol. 2021;35:353-61.

43. Holze F, Caluori TV, Vizeli P, Liechti ME. Safety pharmacology of acute LSD administration in healthy subjects. Psychopharmacology. 13 September 2021. https://doi.org/10.1007/s00213-021-05978-6

44. Hasler F, Bourquin D, Brenneisen R, Bar T, Vollenweider FX. Determination of psilocin and 4-hydroxyindole-3-acetic acid in plasma by HPLC-ECD and 
pharmacokinetic profiles of oral and intravenous psilocybin in man. Pharm Acta Helv. 1997;72:175-84.

45. Dolder PC, Schmid $Y$, Steuer AE, Kraemer T, Rentsch KM, Hammann F, et al. Pharmacokinetics and pharmacodynamics of lysergic acid diethylamide in healthy subjects. Clin Pharmacokinetics. 2017;56:1219-30.

46. Liechti ME, Holze F. Dosing psychedelics and MDMA. Curr Topics Behav Neurosci. 4 November 2021. https://doi.org/10.1007/7854_2021_270

47. Marona-Lewicka D, Chemel BR, Nichols DE. Dopamine D4 receptor involvement in the discriminative stimulus effects in rats of LSD, but not the phenethylamine hallucinogen DOI. Psychopharmacology 2009;203:265-77.

48. Marona-Lewicka D, Thisted RA, Nichols DE. Distinct temporal phases in the behavioral pharmacology of LSD: dopamine D2 receptor-mediated effects in the rat and implications for psychosis. Psychopharmacology 2005;180:427-35.

49. Seifritz E, Baumann P, Muller MJ, Annen O, Amey M, Hemmeter U, et al. Neuroendocrine effects of a $20-\mathrm{mg}$ citalopram infusion in healthy males: a placebocontrolled evaluation of citalopram as 5-HT function probe. Neuropsychopharmacology 1996;14:253-63.

50. Kuypers KP, de la Torre R, Farre M, Yubero-Lahoz S, Dziobek I, Van den Bos W, et al. No evidence that MDMA-induced enhancement of emotional empathy is related to peripheral oxytocin levels or $5-\mathrm{HT}_{1 \mathrm{a}}$ receptor activation. PLoS ONE. 2014;9:e100719.

\section{ACKNOWLEDGEMENTS}

The authors thank Aaron Klaiber, Isidora Avedisian, Fabio Coviello, and Sophie Dierbach for help with conducting the study, Beatrice Vetter for performing the plasma LSD concentration determinations, and Michael Arends for proofreading the paper.

\section{AUTHOR CONTRIBUTIONS}

$\mathrm{FH}, \mathrm{LL}, \mathrm{FM}, \mathrm{PV}$, and MEL designed the research. FH, LL, FM, AMB, IS, PV, SSK, MAR, UD, $\mathrm{KEK}, \mathrm{NV}$, and AE performed the research. FH, UD, KEK, and MEL analyzed the data. FH and MEL wrote the paper with input from all of the other authors. All authors gave final approval to the paper.

\section{FUNDING}

This work was supported by the Swiss National Science Foundation (grant no. 32003B_185111 to MEL). Open access funding provided by University of Basel.

\section{COMPETING INTERESTS}

MEL is a consultant for Mind Medicine, Inc. The other authors declare no competing interests. Knowhow and data associated with this work and owned by the University Hospital Basel were licensed by Mind Medicine, Inc. Mind Medicine, Inc., had no role in financing, planning, or conducting the present study or the present publication.

\section{ADDITIONAL INFORMATION}

Supplementary information The online version contains supplementary material available at https://doi.org/10.1038/s41386-022-01297-2.

Correspondence and requests for materials should be addressed to Matthias E. Liechti.

Reprints and permission information is available at http://www.nature.com/ reprints

Publisher's note Springer Nature remains neutral with regard to jurisdictional claims in published maps and institutional affiliations.

\begin{abstract}
(c) Open Access This article is licensed under a Creative Commons Attribution 4.0 International License, which permits use, sharing, adaptation, distribution and reproduction in any medium or format, as long as you give appropriate credit to the original author(s) and the source, provide a link to the Creative Commons license, and indicate if changes were made. The images or other third party material in this article are included in the article's Creative Commons license, unless indicated otherwise in a credit line to the material. If material is not included in the article's Creative Commons license and your intended use is not permitted by statutory regulation or exceeds the permitted use, you will need to obtain permission directly from the copyright holder. To view a copy of this license, visit http://creativecommons. org/licenses/by/4.0/.
\end{abstract}

(c) The Author(s) 2022 